\title{
VIDEO
}

\section{Digital per-oral cholangioscopy with holmium laser lithotripsy to manage complex biliary calculi}
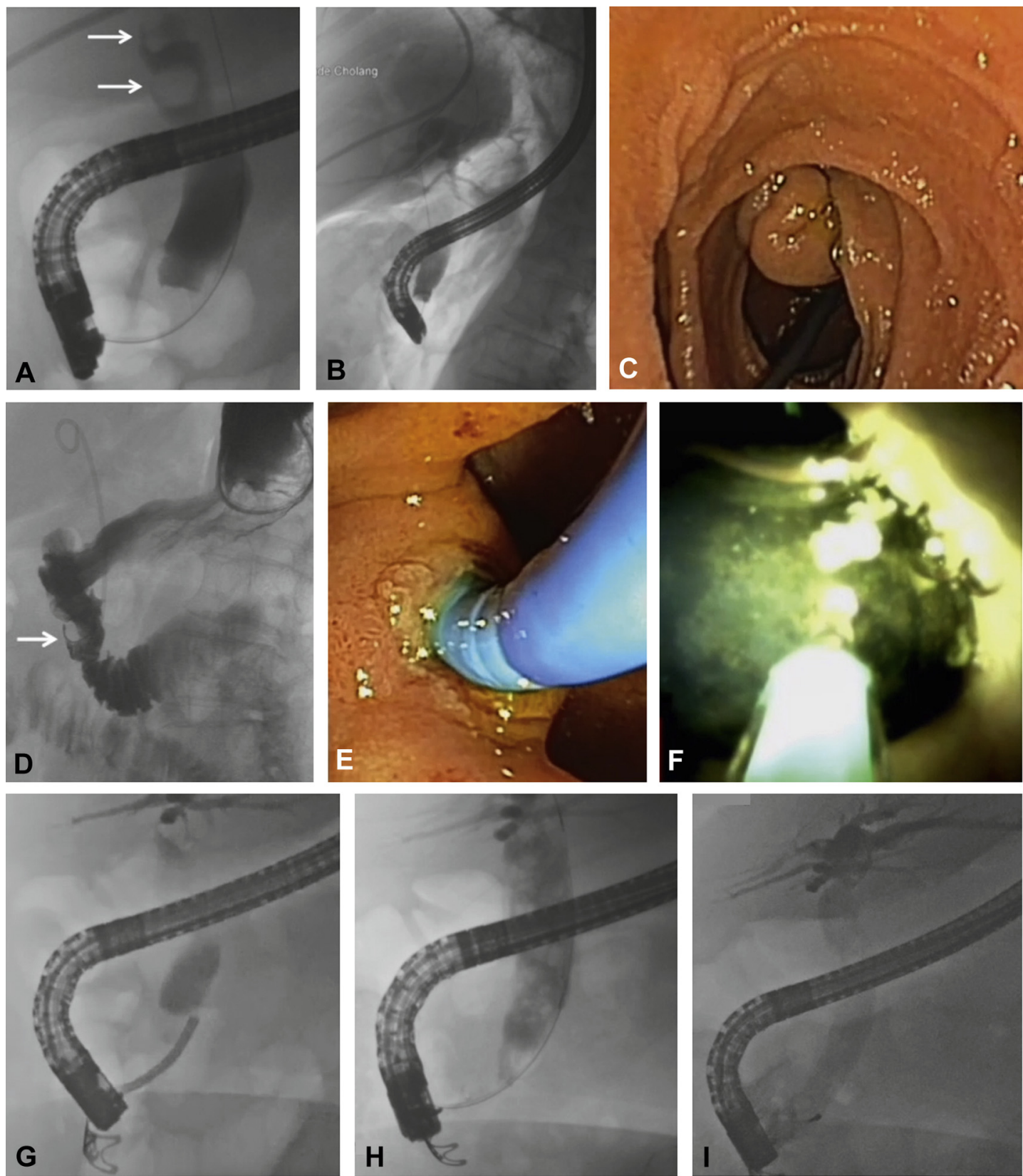

Figure 1. A, Fluoroscopic image taken at ERCP of an 85-year-old woman who presented with cholangitis, demonstrating 2 impacted calculi within the common bile duct (arrows). B, Evidence of retroperitoneal air during the procedure secondary to perforation of a duodenal diverticulum. C, Duodenal perforation closed by successful placement of an over-the-scope-clip. D, Gastrografin study demonstrating no evidence of leak following closure of the duodenal perforation (arrow). E, Digital per-oral cholangioscopy with holmium laser lithotripsy under combined endoscopic (E and $\mathbf{F})$ and fluoroscopic (G) control was used to treat the biliary calculi. This technique was chosen so as not to disrupt the previously treated perforation. H, Fluoroscopic image taken at ERCP demonstrating successful stone fragmentation. I, Fluoroscopic image taken at ERCP demonstrating successful stone clearance. 
We present 3 cases that demonstrate the efficacy and safety of holmium laser lithotripsy in the management of complex biliary calculi. Digital per-oral cholangioscopy performed under fluoroscopic and endoscopic control allows direct visualization of calculi in the intrahepatic and extrahepatic biliary tree. A 200-micron lithotripsy fiber can be advanced down the 1.2-mm channel of the cholangioscope. Visualization is achieved with constant irrigation of normal saline solution $(90-100 \mathrm{~mL} / \mathrm{min})$. Power settings of 10 watts $(10 \mathrm{~Hz}, 1$ joule) are safe to use and allow successful stone fragmentation.

In case 1, a large $(25 \mathrm{~mm})$ and impacted common bile duct calculus was treated successfully with holmium laser lithotripsy. In case 3, holmium laser lithotripsy was used to successfully treat numerous calculi within various intrahepatic right lobe segments under direct endoscopic and fluoroscopic control. The safety of this technique is highlighted in case 2 (Fig. 1). After an iatrogenic duodenal perforation, treated with an over-the-scope clip device, cholangioscopy with holmium laser lithotipsy was used to treat large biliary calculi so as not to disrupt the treated perforation, as can occur with mechanical lithotripsy. Additionally, holmium laser lithotripsy is also less likely to cause bile duct injury when compared with electrohydraulic probes (Video 1, available online at www.VideoGIE.org).

\section{DISCLOSURE}

All authors disclosed no financial relationships relevant to this publication.

Jason Behary, MBBS, Karl Herba, MBBS, FRACP, Philip I. Craig, MBBS, FRACP, PhD, Department of Gastroenterology and Hepatology, St George Hospital and University of New South Wales, Sydney, Australia

Copyright @ 2017 The Authors. Published by Elsevier, Inc. on behalf of the American Society for Gastrointestinal Endoscopy. This is an open access article under the CC BY-NC-ND license (http://creativecommons.org/ licenses/by-nc-nd/4.0/).

http://dx.doi.org/10.1016/j.vgie.2016.12.013 